\title{
Semicopositive linear complementarity systems
}

\author{
Jinglai Shen ${ }^{1}$ and Jong-Shi Pang ${ }^{2, *, \dagger}$ \\ ${ }^{1}$ Department of Mathematics and Statistics, University of Maryland Baltimore County, Baltimore, \\ MD 21250, U.S.A. \\ ${ }^{2}$ Department of Mathematical Sciences, Rensselaer Polytechnic Institute, Troy, NY 12180-3590, U.S.A.
}

\begin{abstract}
SUMMARY
Inspired by the dynamic complementarity problem introduced by Mandelbaum, we define several matrix classes in terms of some integral conditions and discuss their connection with the existing class of strictly semicopositive matrices in linear complementarity theory. Using a time-stepping approximation scheme, we establish the existence of an integrable solution to a class of index-one linear complementarity systems (LCSs) involving these matrices, and that such a solution is 'short-time' unique if the initial state belongs to a semiobservable cone defined in the recent paper (IEEE Trans. Autom. Control 2007, in press). In contrast to the existing well-posedness theory for the LCS, our result is based on a well-known matrix property that has not been used in the LCS literature before. Copyright (C) 2007 John Wiley \& Sons, Ltd.
\end{abstract}

Received 4 April 2006; Revised 7 November 2006; Accepted 28 November 2006

KEY WORDS: linear complementarity systems; observability; time stepping; matrix classes; well-posedness

\section{INTRODUCTION}

Matrix classes play an important role in linear complementarity theory. A variety of such classes have been employed to characterize fundamental solution properties of the linear complementarity problem (LCP), including feasibility, existence (i.e. solvability), and various kinds of uniqueness. For our purpose in this paper, we mention particularly the $P$-matrices, the $S$-matrices, and the (strictly) semicopositive matrices. We refer the reader to the monograph [1] for detailed descriptions of these matrix classes and their roles in LCP theory, see also [2]. (Note on a nomenclature: a (strictly) semicopositive matrix is traditionally called a (strictly)

\footnotetext{
*Correspondence to: Jong-Shi Pang, Department of Mathematical Sciences, Rensselaer Polytechnic Institute, Troy, NY 12180-3590, U.S.A.

†E-mail: pangj@rpi.edu

Contract/grant sponsor: National Science Foundation; contract/grant number: DMS-0508986

Contract/grant sponsor: Focused Research Group; contract/grant number: DMS-0353016
}

Copyright (C) 2007 John Wiley \& Sons, Ltd. 
semimonotone matrix; since these matrices bear a closer connection to a copositive matrix than to a monotone (i.e. positive semidefinite) matrix, we feel that it is more appropriate to use the term 'semicopositivity' than 'semimonotonicity' to reflect the connection; see the precise definition in the next section.)

Being static in nature, the classical LCP is defined on a finite-dimensional Euclidean space and its solutions are vectors in such a space. In recent years, a dynamic version of the LCP, called a linear complementarity system (LCS), has been introduced and studied extensively; the importance of the LCS is well documented in several theses, articles, and surveys [3-11]. In essence, an LCS is a piecewise linear dynamical system defined by a linear time-invariant ordinary differential equation (ODE) that is parameterized by an auxiliary algebraic variable, which is required to be a solution of a finite-dimensional LCP that is in turn linearly coupled with the state of the differential equation. Thus in contrast to the LCP, solutions to the LCS are time-dependent vector functions whose 'regularity' is a principal concern in the study of the well-posedness of the LCS, i.e. the issue of existence and uniqueness of solution trajectories, which is critical to non-smooth and hybrid systems such as the LCS. Needless to say, understanding this issue is the first step to the system analysis and control design of LCSs. Closely related to the LCS is the so-called dynamic complementarity problem (DCP) coined in the unpublished article by Mandelbaum [12]. Although the LCS and DCP are obviously linked, their precise connection has not been formally established. In the process of developing our results in this paper, we will rewrite the LCS in an integral form that will reveal its connection to the DCP in a transparent way; see Lemma 8 and the following remark.

The systematic study of the well-posedness of the LCS began in the two doctoral dissertations $[3,5]$ and continues to date. A brief summary of the state of the art is as follows. In $[5,7,8]$, the authors study this topic under the assumption that all the solutions are locally impulsive smooth, i.e. they are Bohl distributions; sufficient conditions in terms of leading column and row coefficient matrices are derived for the local well-posedness of LCSs. Moreover, local wellposedness conditions expressed in terms of the solvability and uniqueness of the corresponding 'rational complementarity problems' (RCPs) are given in [7] for solutions of Bohl type. (The RCP is a complementarity problem involving rational functions on the complex field and has a close tie with LCSs; see [8] for more discussion.) Based on these results, Çamlibel [3] and Çamlibel and Schumacher [4] show that an index-one LCS has a unique bounded piecewiseBohl solution on any finite time interval for any feasible initial condition. Departing from the class of Bohl solutions, the authors in [13] brought in the classical control-theoretic concept of passivity for the analysis of LCSs. In essence, linear passive complementarity systems are LCSs whose input and output variables satisfy a standard dissipative condition [3]; they form an important subclass of LCSs because of their significant practical values in modelling electrical networks and other physical systems. Under passivity, it was shown in the references that the LCS has a unique $L^{2}$ solution; moreover, the same holds for LCSs that are passifiable by pole shifting. In addition to the well-posedness issue, various system and control theoretic issues have been investigated for the LCS; see [14-21].

Left open in the study of the LCS to date is the question of whether non-passive index-one LCSs possess integrable solutions and whether such solutions are unique. Here, we follow the convention in the recent paper [22] for an index-one system, which is related to but different from what is given in [3]. The main goal of this paper is to address this question formally. Part of the significance of this question is the fact that the class of integrable functions is much broader than that of piecewise-Bohl functions and is more suitable for LCSs that may fail the 
forward-time non-Zeno property [3]. For existence, the main tool we will employ is a timestepping numerical scheme whose convergence will be established by borrowing from the results in the recent paper [23] that pertains to a differential variational inequality, the latter being a generalization of the LCS. For uniqueness, we will impose a certain semiobservability condition on the initial state, the latter property being recently introduced in [19] and subsequently refined in [17] for the study of observability of the LCS. Underlying both issues is a semicopositivity restriction of the LCS and its related reformulations in an integral form that resembles the 'convolution complementarity problem' introduced most recently by Stewart [24].

The remainder of the paper is organized as follows. In Section 2, we review some basic matrix classes in LCP theory and formally define the LCS and the DCP. Section 3 shows solution uniqueness of a DCP with a strictly semicopositive (SSC) defining matrix under non-negative excitation. We next introduce in Section 4 some matrix properties defined in terms of integrals, discuss their equivalences, and establish a key uniqueness result, Proposition 6, which is the cornerstone for solution existence and uniqueness of a special class of the LCS treated in the last section.

\section{PRELIMINARY DISCUSSION}

We begin by summarizing some well-known results for the standard LCP in finite dimensions; see Reference [12] for the review below. Given a vector $q \in \mathfrak{R}^{n}$ and matrix $M \in \mathfrak{R}^{n \times n}$, the LCP $(q, M)$ is to find a vector $z \in \mathfrak{R}^{n}$ such that

$$
0 \leqslant z \perp q+M z \geqslant 0
$$

where the notation $\perp$ means perpendicularity. In this paper, we are particularly interested in the class of SSC matrices $M$ which are defined by the following implication:

$$
[z \geqslant 0 \text { and } z \circ M z \leqslant 0] \Rightarrow z=0
$$

where the notation $\circ$ means the Hadamard product; i.e. $a \circ b$ is the vector whose components are the componentwise products of the two vectors $a$ and $b$. The class of SSC matrices is very broad and includes the class of $P$-matrices, which are characterized by the following implication:

$$
[z \circ M z \leqslant 0] \Rightarrow z=0
$$

The fundamental role of the class of $P$-matrices in LCP theory is the following universal existence and uniqueness result: the $\operatorname{LCP}(q, M)$ has a unique solution for all vectors $q \in \mathfrak{R}^{n}$ if and only if $M$ is a $P$-matrix. There is a similar characterization of the class of SSC matrices. Namely, the LCP $(q, M)$ has a unique solution for all non-negative vectors $q \in \mathfrak{R}^{n}$ if and only if $M$ is SSC. Clearly, a matrix $M$ is SSC if and only if a constant $\sigma>0$ exists such that

$$
\max _{1 \leqslant i \leqslant n} z_{i}(M z)_{i} \geqslant \sigma\|z\|^{2} \quad \forall z \in \mathfrak{R}_{+}^{n}
$$

Another characterization of an SSC matrix is in terms of a completely $S$-property. Specifically, $M \in \mathfrak{R}^{n \times n}$ is an $S$-matrix if a vector $z \geqslant 0$ exists such that $M z>0$. In terms of the LCP, it is easy to see that $M$ is an $S$-matrix if and only if the $\operatorname{LCP}(q, M)$ is feasible (i.e. a vector $z \geqslant 0$ exists satisfying $q+M z \geqslant 0$ ) for all $q \in \mathfrak{R}^{n}$. It is known that $M$ is an SSC matrix if and only if it is completely $S$, i.e. $M$ and all its principal submatrices are $S$-matrices. Moreover, if $M$ is SSC, then so is its transpose. 
For a given $T>0$, which is fixed throughout the paper, let $\operatorname{AC}^{n}[0, T]$ denote the class of absolutely continuous functions $x:[0, T] \rightarrow \mathfrak{R}^{n}$ and let $\mathrm{I}_{+}^{m}[0, T]$ denote the class of integrable functions $u:[0, T] \rightarrow \mathfrak{R}_{+}^{m}$, i.e. $u \geqslant 0$ almost everywhere on $[0, T]$ and $\int_{0}^{T}\|u(t)\| \mathrm{d} t<\infty$. Being absolutely continuous, a function $x \in \mathrm{AC}^{n}[0, T]$ has a derivative $\dot{x}(t)$ for almost all $t \in[0, T]$. Let $\mathrm{AC}_{1+}^{n}[0, T]$ be the subclass of functions $x \in \mathrm{AC}^{n}[0, T]$ with $\dot{x}(t) \geqslant 0$ for almost all $t \in[0, T]$. Defined by a tuple of matrices $(A, B, C, D)$, where $A \in \mathfrak{R}^{n \times n}, B \in \mathfrak{R}^{n \times m}, C \in \mathfrak{R}^{m \times n}$ and $D \in$ $\mathfrak{R}^{m \times m}$, and an initial state $x^{0} \in \mathfrak{R}^{n}$, the $\operatorname{LCS}\left(A, B, C, D, x^{0}\right)$ is to find functions $x \in \mathrm{AC}^{n}[0, T]$ and $u \in \mathrm{I}_{+}^{m}[0, T]$ such that for almost all $t \in[0, T]$,

$$
\begin{aligned}
\dot{x}(t) & =A x(t)+B u(t) \\
0 & \leqslant u(t) \perp C x(t)+D u(t) \geqslant 0 \\
x(0) & =x^{0}
\end{aligned}
$$

Note that in this definition, we restrict the solution class of the state $x$ to be $\operatorname{AC}^{n}[0, T]$ and that of the algebraic variable $u$ to be $\mathrm{I}_{+}^{m}[0, T]$. Such a pair $(x, u)$ is called a weak solution of the LCS. In contrast, Mandelbaum [12] defined the DCP as follows. Let $D^{n}$ denote the space of $n$-dimensional right-continuous-left-limit functions on $[0, \infty)$ and $D_{\wedge}^{n} \equiv\left\{v \in D^{n}: v(0)=0\right.$, $v(t)$ is non-decreasing in $t \geqslant 0\}$. For a given function $q \in D^{n}$ with $q(0) \geqslant 0$, the (continuoustime) $\operatorname{DCP}(q, M)$ is to find $v \in D_{\wedge}^{n}$ such that $q(t)+M v(t) \geqslant 0$ for all $t \geqslant 0$ and $\sum_{i=1}^{n} \int_{0}^{\infty}(q(t)+$ $M v(t))_{i} \mathrm{~d} v_{i}(t)=0$. It is shown in [12] that:

- the DCP $(q, M)$ has a solution for all $q \in D^{n}$ with $q(0) \geqslant 0$ if and only if $M$ is SSC;

- the DCP $(q, M)$ has a unique solution for all $q \in D^{n}$ with $q(0) \geqslant 0$ if and only if the only function $v \in D^{n}$ that is locally of bounded variation with $v(0)=0$ and satisfies

$$
\int_{B}(M v)_{i} \mathrm{~d} v_{i} \leqslant 0 \quad \forall B \in \mathscr{B} \quad \forall i
$$

is the zero function, where $\mathscr{B}$ is the Borel $\sigma$-field in $\mathfrak{R}_{+}$.

While the condition in the last result is more like the $P$-property (2) than the SSC condition (1) (because there is no sign restriction on the function $v$ ), it motivates the definition of several matrix properties in terms of integrals, which we will introduce in Section 4. Incidentally, Mandelbaum has noted that every matrix satisfying the condition in the second result must be a $P$-matrix, but there are $2 \times 2 P$-matrices that fail this condition. Bernard and El Kharroubi [25] also constructed a non-uniqueness example where the defining matrix is a non-negative $P$-matrix.

Subsequently, it will be useful for us to speak of the restricted-time $\operatorname{DCP}(q, M, \widehat{t})$ for a given $q$ in $\operatorname{AC}^{n}[0, T]$ and a scalar $\widehat{t} \in(0, T]$ as the problem of finding a function $v \in \mathrm{AC}_{1+}^{n}[0, T]$ with $v(0)=0$ such that $q(t)+M v(t) \geqslant 0$ for all $t \in[0, \widehat{t}]$ and $\widehat{\int_{0}^{t}} \dot{v}(\tau)^{\mathrm{T}}(q(\tau)+M v(\tau)) \mathrm{d} \tau=0$. Note that since both $\dot{v}(\tau)$ and $q(\tau)+M v(\tau)$ are non-negative on $[0, T]$, the former almost everywhere, we have

$$
\int_{0}^{\widehat{t}} \dot{v}(\tau)^{\mathrm{T}}(q(\tau)+M v(\tau)) \mathrm{d} \tau=0 \Leftrightarrow \int_{0}^{\widehat{t}} \dot{v}(\tau) \circ(q(\tau)+M v(\tau)) \mathrm{d} \tau=0
$$

Besides the obvious difference in the finite upper time $\widehat{t}$, the DCP $(q, M, \widehat{t})$ differs from Mandelbaum's DCP $(q, M)$ in that the former restricts both the input function $q$ and the solution trajectory $v$ to be absolutely continuous; this restriction is consistent with the definition 
of a solution to the LCS which we require to be absolutely continuous. The connection between the restricted-time $\operatorname{DCP}(q, M, \widehat{t})$ and the LCS will be revealed in Lemma 8 .

\section{THE DCP WITH NON-NEGATIVE EXCITATION}

In this section, we consider a related but modified continuous-time DCP that is closely related to the well-posedness of a class of LCSs with index one. This problem, denoted $\operatorname{DCP}_{+}(q, M)$, is defined as follows: for a continuous function $q:[0, \infty) \rightarrow \mathfrak{R}_{+}^{n}$, find a function $v \in \mathrm{AC}_{1+}^{n}[0, \infty)$ with $v(0)=0$ such that $q(t)+M v(t) \geqslant 0$ for all $t \in[0, \infty)$ and

$$
0 \leqslant \dot{v}(t) \perp q(t)+M v(t) \geqslant 0
$$

for almost all $t \in[0, \infty)$. Clearly, with $\dot{v}(t)$ and $q(t)+M v(t)$ being non-negative, the latter complementarity condition is equivalent to the following integral forms:

$$
\int_{0}^{\infty} \dot{v}(\tau)^{\mathrm{T}}(q(\tau)+M v(\tau)) \mathrm{d} \tau=0
$$

and

$$
\int_{0}^{\infty} \dot{v}(\tau) \circ(q(\tau)+M v(\tau)) \mathrm{d} \tau=0
$$

It turns out that the class of SSC matrices characterizes the existence and uniqueness of the zero solution to the $\operatorname{DCP}_{+}(q, M)$ for a class of non-negative continuous functions $q$ that includes all the non-negative affine functions. It should be noted that property (A) stated below for such a function $q$ implies that each element of $q$ is locally of bounded variation when it reaches the zero value. However, such a function is not necessarily absolutely continuous [26, Exercise 5.11].

\section{Proposition 1}

An $n \times n$ matrix $M$ is SSC if and only if for any continuous function $q:[0, \infty) \rightarrow \mathfrak{R}_{+}^{n}$ satisfying the following property for all $i=1, \ldots, n$ :

(A) if $q_{i}\left(t_{*}\right)=0$ for some $t_{*} \geqslant 0$, then $\varepsilon_{i}>0$ exists such that $q_{i}(t)$ is non-decreasing for all $t \in\left[t_{*}, t_{*}+\varepsilon_{i}\right]$.

$v(t) \equiv 0$ is the unique solution to the $\mathrm{DCP}_{+}(q, M)$ on $[0, \infty)$.

\section{Proof}

If $M$ is not SSC, then there exists a non-zero vector $\widehat{v} \geqslant 0$ such that $\widehat{v}_{i}(M \widehat{v})_{i} \leqslant 0$ for all $i=$ $1, \ldots, n$. Let $q(t) \equiv \max (0,-(M \widehat{v})) t$ and $v(t) \equiv t \widehat{v}$ for all $t \geqslant 0$. It is easy to show that $v(t)$ is a nonzero solution of the $\mathrm{DCP}_{+}(q, M)$. This establishes the 'if' statement.

For the 'only if' statement, it suffices to show the uniqueness of the solution. We prove this by induction on $n$. Let $M$ be an SSC matrix of order $n$, let $q(t)$ be a non-negative continuous function satisfying property (A), and let $v(t)$ be a solution of the $\operatorname{DCP}_{+}(q, M)$. When $n=1, M$ is a positive scalar $m$ and $v(t)$ is a real-valued function. Thus for any $t \in[0, \infty)$,

$$
0=\int_{0}^{t} \dot{v}(\tau)(q(\tau)+M v(\tau)) \mathrm{d} \tau=\int_{0}^{t} \dot{v}(\tau) q(\tau) \mathrm{d} \tau+\frac{m}{2} v^{2}(t) \geqslant \frac{m}{2} v^{2}(t)
$$

This implies that $v(t) \equiv 0$ everywhere. To prove the uniqueness for general $n$, we first show that (a) an $\varepsilon>0$ exists such that $v(t) \equiv 0$ for all $t \in[0, \varepsilon]$. 
For notational convenience, let $w(t):=q(t)+M v(t)$. Clearly, $w(t)$ is non-negative and continuous for all $t \geqslant 0$. Consider two cases:

Case 1: $q_{i}(0)>0$ for some index $i$. In this case, we have $w_{i}(0)=q_{i}(0)>0$. Hence by continuity, an $\varepsilon>0$ exists such that $w_{i}(t)>0$ on $[0, \varepsilon]$. Therefore, $\dot{v}_{i}(t)=0$ for almost all $t \in[0, \varepsilon]$, which implies, since $v_{i}(0)=0$, that $v_{i}(t)=0$ on $[0, \varepsilon]$. Letting $\theta=\{1, \ldots, n\} \backslash\{i\}$, we deduce that the $n$-dimensional $\mathrm{DCP}_{+}(q, M)$ is reduced to the $(n-1)$-dimensional $\mathrm{DCP}_{+}\left(q_{\theta}, M_{\theta \theta}\right)$ on the interval $[0, \varepsilon]$ :

$$
0 \leqslant \dot{v}_{\theta}(t) \perp q_{\theta}(t)+M_{\theta \theta} v_{\theta}(t) \geqslant 0
$$

Since $M_{\theta \theta}$ remains SSC and $q_{\theta}(t)$ is non-negative and continues to satisfy condition (A), the induction hypothesis yields $v_{\theta}(t)=0$, and thus $v(t) \equiv 0$, on $[0, \varepsilon]$.

Case 2: $q(0)=0$. By (A), there exists $\varepsilon>0$ such that $q(t)$ is non-decreasing on $[0, \varepsilon]$. Suppose that this $\varepsilon$ fails the claim (a). Then $w(t) \supsetneqq 0$ for all $t \in(0, \varepsilon]$. Indeed, if $w\left(t^{\prime}\right)=0$ for some $t^{\prime}$ in the latter interval, then $M v\left(t^{\prime}\right) \leqslant 0$, which yields $v\left(t^{\prime}\right)=0$ by the SSC property of $M$. In turn, since $v$ is non-decreasing, we deduce that $v \equiv 0$ on $\left[0, t^{\prime}\right]$. Therefore, for any $t_{*} \in(0, \varepsilon)$, there exist an index $i$ and two scalars $t_{1}$ and $t_{2}$ such that $0 \leqslant t_{1}<t_{*}<t_{2} \leqslant \varepsilon$ and $w_{i}(t)>0$ on $\left[t_{1}, t_{2}\right] \subseteq[0, \varepsilon]$. Hence, $\dot{v}_{i}(t) \equiv 0$ on $\left[t_{1}, t_{2}\right]$ and the $\mathrm{DCP}_{+}(q, M)$ on the same interval becomes

$$
0 \leqslant \dot{v}_{\theta}(t) \perp w_{\theta}\left(t_{1}\right)+\left[q(t)-q\left(t_{1}\right)\right]_{\theta}+M_{\theta \theta} \int_{t_{1}}^{t} \dot{v}_{\theta}(\tau) \mathrm{d} \tau \geqslant 0
$$

where $\theta=\{1, \ldots, n\} \backslash\{i\}$. By letting $u(s)=\int_{t_{1}}^{s+t_{1}} \dot{v}_{\theta}(\tau) \mathrm{d} \tau$ and $\widetilde{q}(s)=w_{\theta}\left(t_{1}\right)+\left[q\left(s+t_{1}\right)-q\left(t_{1}\right)\right]_{\theta}$ for $s \in\left[0, t_{2}-t_{1}\right]$, the original $\mathrm{DCP}_{+}(q, M)$ is reduced to the $(n-1)$-dimensional $\mathrm{DCP}_{+}\left(\widetilde{q}, M_{\theta \theta}\right)$ on the interval $\left[0, t_{2}-t_{1}\right]$ :

$$
0 \leqslant \dot{u}(s) \perp \widetilde{q}(s)+M_{\theta \theta} u(s) \geqslant 0
$$

Noticing that the latter DCP of reduced dimension continues to satisfy all the properties of the original DCP, we deduce by induction hypothesis that $u(s) \equiv 0$ on $\left[0, t_{2}-t_{1}\right]$; thus $v(t) \equiv v\left(t_{1}\right)$ on $\left[t_{1}, t_{2}\right]$. Moreover, for any sufficiently small $t_{0}>0$, a covering argument on the compact interval $\left[t_{0}, \varepsilon\right]$ shows that $v(t) \equiv v\left(t_{0}\right)$ for all $t \in\left[t_{0}, \varepsilon\right]$. Hence, $v(\varepsilon)=v\left(t_{0}\right)$. By the continuity of $v(t)$, it follows that $v(\varepsilon)=\lim _{t_{0} \downarrow} v\left(t_{0}\right)=0$. Hence $v(t) \equiv 0$ on $[0, \varepsilon]$. This is a contraction; thus (a) holds.

To show that $v \equiv 0$ on $[0, \infty)$, let $t_{*} \equiv \sup \{t \geqslant 0: v \equiv 0$ on $[0, t]\} \geqslant \varepsilon$. If $t_{*}<\infty$, then shifting time as done in the above proof and applying (a), we easily obtain a contradiction.

An important consequence of the above proposition is related to the solution uniqueness for a class of LCSs with $D=0$. We let $I_{n}$ denote the identity matrix of order $n$.

\section{Corollary 2}

If $C B$ is SSC and $x^{0}$ is feasible, i.e. $C x^{0} \geqslant 0$, then $\left(x\left(t, x^{0}\right), u\left(t, x^{0}\right)\right) \equiv\left(\mathrm{e}^{\lambda t} x^{0}, 0\right)$ is the unique solution pair to the $\operatorname{LCS}\left(\lambda I_{n}, B, C, D\right)$ on $[0, \infty)$ for all scalars $\lambda$.

\section{Proof}

It is observed in [3] that for any real $\rho$, if $\left(x\left(t, x^{0}\right), u\left(t, x^{0}\right)\right)$ is a weak solution pair of the $\operatorname{LCS}(A, B, C, D)$ for a feasible initial condition $x^{0}$, then $\left(\mathrm{e}^{\rho t} x\left(t, x^{0}\right), \mathrm{e}^{\rho t} u\left(t, x^{0}\right)\right)$ is a weak solution pair of the $\operatorname{LCS}\left(A+\rho I_{n}, B, C, D\right)$. Therefore, the $\operatorname{LCS}\left(\lambda I_{n}, B, C, 0\right)$ has a unique weak solution if 
and only if the $\operatorname{LCS}(0, B, C, 0)$ does. The latter LCS is

$$
\dot{x}=B u, \quad 0 \leqslant u \perp C x \geqslant 0
$$

whose integral form is

$$
0 \leqslant u(t) \perp C x^{0}+C B \int_{0}^{t} u(\tau) \mathrm{d} \tau \geqslant 0
$$

Since $C x^{0} \geqslant 0$ and $C B$ is SSC, by Proposition 1 , the unique weak solution pair to the $\operatorname{LCS}(0$, $B, C, 0)$ on $[0, \infty)$ is given by the constant $\left(x^{0}, 0\right)$.

\section{MATRIX CLASSES}

Motivated by the integral form of the complementarity condition in both DCPs, we introduce three properties of a matrix $M \in \mathfrak{R}^{n \times n}$ by casting expression (3) in an integral form, which we call SSC of types I-III, respectively:

I scalars $\sigma>0$ and $\bar{t} \in(0, T]$ exist such that for all $v \in \mathrm{AC}_{1+}^{n}[0, T]$ with $v(0)=0$, the following condition holds for all $t \in[0, \bar{t}]$ :

$$
\max _{1 \leqslant i \leqslant n}\left\{\int_{0}^{t} \dot{v}_{i}(\tau)(M v(\tau))_{i} \mathrm{~d} \tau\right\} \geqslant \sigma\|v(t)\|^{2}
$$

II for each $v \in \mathrm{AC}_{1+}^{n}[0, T]$ with $v(0)=0$, scalars $\sigma_{v}>0$ and $t_{v} \in(0, T]$ exist such that for all $t \in\left[0, t_{v}\right]$, the following condition holds:

$$
\max _{1 \leqslant i \leqslant n}\left\{\int_{0}^{t} \dot{v}_{i}(\tau)(M v(\tau))_{i} \mathrm{~d} \tau\right\} \geqslant \sigma_{v}\|v(t)\|^{2}
$$

III for each $v \in \mathrm{AC}_{1+}^{n}[0, T]$ with $v(0)=0$, a scalar $t_{v} \in(0, T]$ exists such that for any $t \in\left[0, t_{v}\right]$,

$$
\int_{0}^{t} \dot{v}(\tau) \circ M v(\tau) \mathrm{d} \tau \leqslant 0 \Rightarrow v=0 \quad \text { on }[0, t]
$$

The above three conditions are all 'short-time' in nature in the sense that they pertain to the existence of times, $\bar{t}$ in type I and $t_{v}$ in II and III, for which the conditions are valid. Note the distinctive roles of these times $\bar{t}$ and $t_{v}$, the former is independent of the functions $v$, whereas the latter depends on the particular function $v$. To be consistent with these matrix types, we call a matrix $M$ satisfying the vector condition (3) SSC of type 0 . We state two obvious facts about the four types of SSC conditions.

- If a matrix is SSC of any of the above types, then all its principal submatrices are of the same type; hence these matrix properties are complete.

- Clearly, type I $\Rightarrow \mathrm{II} \Rightarrow \mathrm{III} \Rightarrow 0$. To see the last implication, suppose III holds. Let $v^{0}$ be an arbitrary non-negative vector satisfying $v^{0} \circ M v^{0} \leqslant 0$. Define the function $v(t) \equiv t v^{0}$, which clearly belongs to $\operatorname{AC}_{1+}^{n}[0, T]$ because $v^{0} \geqslant 0$. Since

$$
\int_{0}^{t} \dot{v}(\tau) \circ M v(\tau) \mathrm{d} \tau=\frac{t^{2}}{2}\left[v^{0} \circ M v^{0}\right] \leqslant 0
$$

condition III yields $v^{0}=0$. 
We next identify several classes of matrices that fall into type I. Such matrices include the symmetric positive definite ones.

\section{Proposition 3}

A matrix $M \in \mathfrak{R}^{n \times n}$ of any one of the following classes is SSC of type I:

(a) $M$ is non-negative with positive diagonal entries;

(b) $M$ is symmetric and strictly copositive;

(c) $M$ is symmetric positive definite;

(d) $M$ is symmetric and SSC of type 0 .

Moreover, if $M$ is SSC of type I, so is $D M E$, where $D$ and $E$ are any diagonal matrices with positive diagonal entries.

Proof

We show (a) first. Letting $m_{i j}$ denote the $i j$-entry of $M$, we see that for all $v \in \mathrm{AC}_{1+}^{n}[0, T]$ with $v(0)=0$ and some $T>0$

$$
\max _{1 \leqslant i \leqslant n}\left\{\int_{0}^{t} \dot{v}_{i}(\tau)(M v(\tau))_{i} \mathrm{~d} \tau\right\} \geqslant \frac{1}{n} \int_{0}^{t} \dot{v}^{\mathrm{T}}(\tau) M v(\tau) \mathrm{d} \tau \geqslant \frac{1}{2 n} \sum_{i=1}^{n} m_{i i} v_{i}^{2}(t) \geqslant \frac{\min _{i}\left(m_{i i}\right)}{2 n}\|v(t)\|^{2}
$$

holds for all $t \in[0, T]$, where we use the non-negativity of $m_{i j}$, $\dot{v}(t)$ and $v(t)$ as well as the fact that the diagonal entry $m_{i i}>0$ for all $i=1, \ldots, n$. This implies that $M$ belongs to the class of type I matrices.

In case (b), we have again, for all $v \in \mathrm{AC}_{1+}^{n}[0, T]$ with $v(0)=0$ and some $T>0$,

$$
\max _{1 \leqslant i \leqslant n}\left\{\int_{0}^{t} \dot{v}_{i}(\tau)(M v(\tau))_{i} \mathrm{~d} \tau\right\} \geqslant \frac{1}{n} \int_{0}^{t} \dot{v}^{\mathrm{T}}(\tau) M v(\tau) \mathrm{d} \tau
$$

holds for all $t \in[0, T]$. Due to the symmetry of $M$ and the product rule for the absolutely continuous functions $v_{i}(t)$ and $v_{j}(t)[27$, Exercise 3.35]

$$
\int_{0}^{t}\left[\dot{v}_{i}(\tau) v_{j}(\tau)+\dot{v}_{j}(\tau) v_{i}(\tau)\right] \mathrm{d} \tau=v_{i}(t) v_{j}(t)
$$

we further have

$$
\int_{0}^{t} \dot{v}^{\mathrm{T}}(\tau) M v(\tau) \mathrm{d} \tau=\frac{1}{2} v^{\mathrm{T}}(t) M v(t)
$$

Since $M$ is strictly copositive, i.e. $z^{\mathrm{T}} M z>0$ for all $0 \neq z \in \mathfrak{R}_{+}^{n}$, a positive scalar $\mu$ exists such that $z^{\mathrm{T}} M z \geqslant \mu\|z\|^{2}$ for all $z \in \mathfrak{R}_{+}^{n}$. Therefore, we have, for all $t \in[0, T]$,

$$
\max _{1 \leqslant i \leqslant n}\left\{\int_{0}^{t} \dot{v}_{i}(\tau)(M v(\tau))_{i} \mathrm{~d} \tau\right\} \geqslant \frac{\mu}{2 n}\|v(t)\|^{2}
$$

This shows that $M$ is of type $\mathrm{I}$.

To show the desired result under condition (c), we note that a positive definite matrix must be strictly copositive. If it is also symmetric, then it satisfies condition (b). Thus, it is of type I. Furthermore, if $M$ is SSC and symmetric, then it is strictly copositive [1, Proposition 3.9.14]. Hence, the desired result holds under condition (d). 
To show the last statement, it suffices to prove that if $M$ of type I, so are $D M$ and $M D$ for any diagonal matrix $D=\operatorname{diag}\left(\lambda_{1}, \ldots, \lambda_{n}\right)$ with $\lambda_{i}>0$. We show that $D M$ is of type I first. Let $\widetilde{M}=D M$ and thus $M=D^{-1} \widetilde{M}$. Since $M$ is of type I, positive scalars $T$ and $\sigma$ exist such for all $v \in \mathrm{AC}_{1+}^{n}[0, T]$ with $v(0)=0$,

$$
\max _{1 \leqslant i \leqslant n}\left\{\int_{0}^{t} \dot{v}_{i}(\tau)\left(D^{-1} \tilde{M} v(\tau)\right)_{i} \mathrm{~d} \tau\right\}=\max _{1 \leqslant i \leqslant n}\left\{\lambda_{i}^{-1} \int_{0}^{t} \dot{v}_{i}(\tau)(\tilde{M} v(\tau))_{i} \mathrm{~d} \tau\right\} \geqslant \sigma\|v(t)\|^{2}
$$

On the other hand, for all $v \in \mathrm{AC}_{1+}^{n}[0, T]$ with $v(0)=0$ and $t \in[0, T]$,

$$
\max _{1 \leqslant i \leqslant n}\left\{\lambda_{i}^{-1} \int_{0}^{t} \dot{v}_{i}(\tau)(\widetilde{M} v(\tau))_{i} \mathrm{~d} \tau\right\} \leqslant \max _{1 \leqslant i \leqslant n}\left(\lambda_{i}^{-1}\right) \max _{1 \leqslant i \leqslant n}\left\{\int_{0}^{t} \dot{v}_{i}(\tau)(\tilde{M} v(\tau))_{i} \mathrm{~d} \tau\right\}
$$

where we make use of the positivity of $\lambda_{i}$. Consequently,

$$
\max _{1 \leqslant i \leqslant n}\left\{\int_{0}^{t} \dot{v}_{i}(\tau)(\tilde{M} v(\tau))_{i} \mathrm{~d} \tau\right\} \geqslant \frac{\sigma}{\max _{i}\left(\lambda_{i}^{-1}\right)}\|v(t)\|^{2}
$$

Hence, $D M$ is of type I. Similarly, for $\widetilde{M}=M D$, we have, for all $v \in \mathrm{AC}_{1+}^{n}[0, T]$ with $v(0)=0$ and $t \in[0, T]$,

$$
\max _{1 \leqslant i \leqslant n}\left\{\int_{0}^{t}\left(\lambda_{i} \dot{v}_{i}(\tau)\right)(\widetilde{M} v(\tau))_{i} \mathrm{~d} \tau\right\} \geqslant \sigma\|D v(t)\|^{2} \geqslant \sigma \min _{i}\left(\lambda_{i}^{2}\right)\|v(t)\|^{2}
$$

Noticing that

$$
\max _{1 \leqslant i \leqslant n}\left\{\int_{0}^{t}\left(\lambda_{i} \dot{v}_{i}(\tau)\right)(\widetilde{M} v(\tau))_{i} \mathrm{~d} \tau\right\} \leqslant \max _{1 \leqslant i \leqslant n}\left(\lambda_{i}\right) \max _{1 \leqslant i \leqslant n}\left\{\int_{0}^{t} \dot{v}_{i}(\tau)(\tilde{M} v(\tau))_{i} \mathrm{~d} \tau\right\}
$$

we obtain

$$
\max _{1 \leqslant i \leqslant n}\left\{\int_{0}^{t} \dot{v}_{i}(\tau)(\tilde{M} v(\tau))_{i} \mathrm{~d} \tau\right\} \geqslant \frac{\sigma \min _{i}\left(\lambda_{i}^{2}\right)}{\max _{i}\left(\lambda_{i}\right)}\|v(t)\|^{2}
$$

which leads to the desired result.

It turns out that all the four SSC types 0, I-III are equivalent for matrices of order 2, and are further equivalent to the 'short-time uniqueness' of AC solutions of the DCP with non-negative AC input functions. To prepare for the proof, we note that all the $2 \times 2$ SSC matrices can be expressed in one of the following two forms by suitable scaling and variable permutation:

$$
\left[\begin{array}{ll}
1 & \alpha \\
\beta & 1
\end{array}\right], \quad \beta \geqslant 0, \quad\left[\begin{array}{cc}
1 & -\alpha \\
-\beta & 1
\end{array}\right], \quad \alpha>0, \quad \beta>0, \quad 0<\alpha \beta<1
$$

\section{Proposition 4}

The following implications hold for any SSC matrix $M \in \mathfrak{R}^{2 \times 2}$ :

$$
M \text { is of type I } \Leftrightarrow M \text { is of type II } \Leftrightarrow M \text { is of type III } \Leftrightarrow M \text { is of type } 0
$$

\section{Proof}

To prove this statement, it suffices to show every $2 \times 2$ SSC matrix of type 0 must be of type I. Assume the contrary. Then there exists a sequence of positive scalars $\left\{\sigma_{k}\right\}$ converging to zero 
such that for each $k$, a $v^{k}(t) \in \mathrm{AC}_{1+}^{2}[0, T]$ with $v^{k}(0)=0$ exists satisfying

$$
\max _{i=1,2}\left\{\int_{0}^{t_{k}} \dot{v}_{i}^{k}(\tau)\left(M v^{k}(\tau)\right)_{i} \mathrm{~d} \tau\right\}<\sigma_{k}\left\|v^{k}\left(t_{k}\right)\right\|^{2}
$$

for some $t_{k}>0$. It is clear that $v^{k}\left(t_{k}\right) \neq 0$ for all $k$. Without loss of generality, we may assume that the limit

$$
\lim _{k \rightarrow \infty} \frac{v^{k}\left(t_{k}\right)}{\left\|v^{k}\left(t_{k}\right)\right\|}=v^{*}
$$

exists, which must be non-negative and satisfies $\left(v_{1}^{*}\right)^{2}+\left(v_{2}^{*}\right)^{2}=1$. Consider the first case where

$$
M=\left[\begin{array}{ll}
1 & \alpha \\
\beta & 1
\end{array}\right], \quad \beta \geqslant 0
$$

Since

$$
\int_{0}^{t_{k}} \dot{v}_{1}^{k}(\tau)\left[v_{1}^{k}(\tau)+\alpha v_{2}^{k}(\tau)\right] \mathrm{d} \tau<\sigma_{k}\left\|v^{k}\left(t_{k}\right)\right\|_{2}^{2}, \quad \int_{0}^{t_{k}} \dot{v}_{2}^{k}(\tau)\left[\beta v_{1}^{k}(\tau)+v_{2}^{k}(\tau)\right] \mathrm{d} \tau<\sigma_{k}\left\|v^{k}\left(t_{k}\right)\right\|_{2}^{2}
$$

we have, from the second inequality and the non-negativity of $\beta, \dot{v}^{k}, v^{k}$,

$$
\int_{0}^{t_{k}} \dot{v}_{2}^{k}(\tau) v_{2}^{k}(\tau) \mathrm{d} \tau=\frac{1}{2}\left(v_{2}^{k}\left(t_{k}\right)\right)^{2} \leqslant \int_{0}^{t_{k}} \dot{v}_{2}^{k}(\tau)\left[\beta v_{1}^{k}(\tau)+v_{2}^{k}(\tau)\right] \mathrm{d} \tau<\sigma_{k}\left\|v^{k}\left(t_{k}\right)\right\|_{2}^{2}
$$

Hence, letting $k \rightarrow \infty$, we get $v_{2}^{*}=0$ which further implies $v_{1}^{*}=1$. For $\alpha \geqslant 0$, similarly we have $v_{1}^{*}=0$, which is a contradiction. If $\alpha<0$, we have, by integration by parts,

$$
\begin{aligned}
\int_{0}^{t_{k}} \dot{v}_{1}^{k}(\tau)\left[v_{1}^{k}(\tau)+\alpha v_{2}^{k}(\tau)\right] \mathrm{d} \tau & =\frac{1}{2}\left(v_{1}^{k}\left(t_{k}\right)\right)^{2}+\alpha v_{1}^{k}\left(t_{k}\right) v_{2}^{k}\left(t_{k}\right)-\alpha \int_{0}^{t_{k}} v_{1}^{k}(\tau) \dot{v}_{2}^{k}(\tau) \mathrm{d} \tau \\
& \geqslant \frac{1}{2}\left(v_{1}^{k}\left(t_{k}\right)\right)^{2}+\alpha v_{1}^{k}\left(t_{k}\right) v_{2}^{k}\left(t_{k}\right)
\end{aligned}
$$

Hence, using the first inequality and letting $k \rightarrow \infty$, we get $\frac{1}{2}\left(v_{1}^{*}\right)^{2}+\alpha v_{1}^{*} v_{2}^{*} \leqslant 0$. But this contradicts $\left(v_{1}^{*}, v_{2}^{*}\right)=(1,0)$. This shows that $M$ must be of type I.

Consider the next case where

$$
M=\left[\begin{array}{cc}
1 & -\alpha \\
-\beta & 1
\end{array}\right], \quad \alpha>0, \quad \beta>0, \quad 0<\alpha \beta<1
$$

In this case, we have

$$
\int_{0}^{t_{k}} \dot{v}_{1}^{k}(\tau) v_{1}^{k}(\tau) \mathrm{d} \tau-\sigma_{k}\left\|v^{k}\left(t_{k}\right)\right\|_{2}^{2}<\alpha \int_{0}^{t_{k}} \dot{v}_{1}^{k}(\tau) v_{2}^{k}(\tau) \mathrm{d} \tau
$$

and

$$
\int_{0}^{t_{k}} \dot{v}_{2}^{k}(\tau) v_{2}^{k}(\tau) \mathrm{d} \tau-\sigma_{k}\left\|v^{k}\left(t_{k}\right)\right\|_{2}^{2}<\beta \int_{0}^{t_{k}} \dot{v}_{2}^{k}(\tau) v_{1}^{k}(\tau) \mathrm{d} \tau
$$

Hence,

$$
\frac{1}{2 \alpha}\left[v_{1}^{k}\left(t_{k}\right)\right]^{2}+\frac{1}{2 \beta}\left[v_{2}^{k}\left(t_{k}\right)\right]^{2}-\left(\frac{1}{\alpha}+\frac{1}{\beta}\right) \sigma_{k}\left\|v^{k}\left(t_{k}\right)\right\|_{2}^{2}<v_{1}^{k}\left(t_{k}\right) v_{2}^{k}\left(t_{k}\right)
$$


Letting $k \rightarrow \infty$, we obtain

$$
\frac{1}{2 \alpha}\left(v_{1}^{*}\right)^{2}+\frac{1}{2 \beta}\left(v_{2}^{*}\right)^{2} \leqslant v_{1}^{*} v_{2}^{*}
$$

It is clear that $v_{1}^{*}$ and $v_{2}^{*}$ must be both positive to satisfy the inequality. Moreover,

$$
\frac{1}{2 \alpha}\left(v_{1}^{*}\right)^{2}+\frac{1}{2 \beta}\left(v_{2}^{*}\right)^{2} \geqslant 2 \sqrt{\frac{1}{4 \alpha \beta}} v_{1}^{*} v_{2}^{*} \geqslant \sqrt{\frac{1}{\alpha \beta}} v_{1}^{*} v_{2}^{*}>v_{1}^{*} v_{2}^{*}
$$

This is a contradiction.

Next we establish the relation between the matrix classes discussed above and the solution of the restricted-time $\operatorname{DCP}(q, M, \widehat{t})$ defined in Section 2.

\section{Proposition 5}

The following statements hold:

(a) If $M$ is of type I, then scalars $\bar{\sigma}>0$ and $\bar{t} \in(0, T]$ exist such that for any $q \in \operatorname{AC}^{n}[0, T]$, any solution $v$ of the $\operatorname{DCP}(q, M, \bar{t})$ satisfies

$$
\sup _{t \in[0, \bar{t}]}\|v(t)\|=\|v(\bar{t})\| \leqslant \bar{\sigma} \sup _{t \in[0, \bar{t}]}\|\max (0,-q(t))\|
$$

(b) If $M$ is of type III, then for any non-negative $q \in \mathrm{AC}^{n}[0, T]$, any solution $v$ of the DCP $\left(q, M, t_{q, v}\right)$ for some $t_{q, v}>0$ must satisfy $v=0$ on $\left[0, t_{q, v}^{\prime}\right]$ for some $t_{q, v}^{\prime} \in\left(0, t_{q, v}\right]$.

\section{Proof}

To prove statement (a), let $M$ be of type I and let $\sigma$ and $\bar{t}$ be two positive scalars associated with this type. For any solution $v$ of the $\operatorname{DCP}(q, M, \bar{t})$, where $q \in \operatorname{AC}^{n}[0, T]$, we have

$$
0=\int_{0}^{\bar{t}} \dot{v}(\tau) \circ(q(\tau)+M v(\tau)) \mathrm{d} \tau
$$

which yields

$$
\begin{aligned}
\sigma\|v(\bar{t})\|^{2} & \leqslant \max _{1 \leqslant i \leqslant n} \int_{0}^{\bar{t}} \dot{v}_{i}(\tau)(M v(\tau))_{i} \mathrm{~d} \tau=\max _{1 \leqslant i \leqslant n} \int_{0}^{\bar{t}} \dot{v}_{i}(\tau)\left(-q_{i}(\tau)\right) \mathrm{d} \tau \\
& \leqslant \max _{1 \leqslant i \leqslant n}\left[\int_{0}^{\bar{t}} \dot{v}_{i}(\tau) \mathrm{d} \tau\right] \sup _{t \in[0, \bar{t}]}\|\max (0,-q(t))\| \quad \text { because } \dot{v} \geqslant 0 \text { almost everywhere } \\
& \leqslant\|v(\bar{t})\| \sup _{t \in[0, \bar{t}]}\|\max (0,-q(t))\| \quad \text { because } v(0)=0
\end{aligned}
$$

Hence,

$$
\sup _{t \in[0, \bar{t}]}\|v(t)\|=\|v(\bar{t})\| \leqslant \sigma^{-1} \sup _{t \in[0, \bar{t}]}\|\max (0,-q(t))\|
$$

where the equality holds because $v(\bar{t}) \geqslant v(t) \geqslant 0$ for all $t \in[0, \bar{t}]$. 
To prove statement (b), consider a matrix $M$ of type III, and let $\left(q, v, t_{q, v}\right)$ be as given. Let $t_{v}>0$ be the time prescribed by the matrix $M$. Let $t_{q, v}^{\prime} \equiv \min \left(t_{q, v}, t_{v}\right)$. We have,

$$
\int_{0}^{t_{q, v}^{\prime}} \dot{v}(\tau) \circ M v(\tau) \mathrm{d} \tau \leqslant \int_{0}^{t_{q, v}^{\prime}} \dot{v}(\tau) \circ(q(\tau)+M v(\tau)) \mathrm{d} \tau \leqslant \int_{0}^{t_{q, v}} \dot{v}(\tau) \circ(q(\tau)+M v(\tau)) \mathrm{d} \tau=0
$$

where the two inequalities hold because the functions within the integrals are non-negative. By the type III condition, it follows that $v=0$ on $\left[0, t_{q, v}^{\prime}\right]$. This completes the proof of statement (b).

The result below is the key to the solution uniqueness for the LCS to be studied in the next section.

\section{Proposition 6}

Let $M \in \mathfrak{R}^{m \times m}$ and $\varepsilon>0$ be given. Let $f:[0, \varepsilon] \rightarrow \mathfrak{R}_{+}^{m}$ be a non-negative absolutely continuous function and $\Phi:[0, \varepsilon] \times \mathrm{I}^{m}[0, \varepsilon] \rightarrow \mathfrak{R}^{m}$ be such that $\Phi(\cdot, u)$ is an absolutely continuous function in the first argument for every $u \in \mathrm{I}^{m}[0, \varepsilon]$ and that for some scalar $\mu>0,\|\Phi(t, u)\| \leqslant$ $\mu t\left\|\int_{0}^{t} u(\tau) \mathrm{d} \tau\right\|$ for all $t \in[0, \varepsilon]$ and all $u \in \mathrm{I}_{+}^{m}[0, \varepsilon]$. The following two statements hold:

(a) If $M$ is SSC of type II, then for every integrable function $u(t)$ satisfying

$$
0 \leqslant u(t) \perp f(t)+\Phi(t, u)+M \int_{0}^{t} u(\tau) \mathrm{d} \tau \geqslant 0, \quad \text { for almost all } t \in[0, \varepsilon]
$$

there exists $\varepsilon_{u} \in(0, \varepsilon]$ such that $u=0$ almost everywhere on $\left[0, \varepsilon_{u}\right]$.

(b) If $M$ is SSC of type I, then there exists $\varepsilon^{\prime} \in(0, \varepsilon]$ such that for every integrable function $u(t)$ satisfying (4), it holds that $u=0$ almost everywhere on $\left[0, \varepsilon^{\prime}\right]$.

\section{Proof}

Let $u(t)$ be an arbitrary integrable function satisfying (4). Define $v(t) \equiv \int_{0}^{t} u(\tau) \mathrm{d} \tau$, which belongs to $\mathrm{AC}_{1+}^{m}[0, \varepsilon]$. Since

$$
\int_{0}^{t} u_{i}(s)[f(s)+M v(s)+\Phi(s, u)]_{i} \mathrm{~d} s=0
$$

for all $t \in[0, \varepsilon]$ and all $i=1, \ldots, m$, we have, by the non-negativity of $f(t), v(t)$ and $\dot{v}(t)$,

$$
\begin{aligned}
0 & \geqslant-\int_{0}^{t} \dot{v}_{i}(\tau) f_{i}(\tau) \mathrm{d} \tau=\int_{0}^{t}\left\{\dot{v}_{i}(\tau)(M v(\tau))_{i}+\dot{v}_{i}(\tau) \Phi(\tau, u)_{i}\right\} \mathrm{d} \tau \\
& \geqslant \int_{0}^{t}\left\{\dot{v}_{i}(\tau)(M v(\tau))_{i}-\dot{v}_{i}(\tau)\left|\Phi(\tau, u)_{i}\right|\right\} \mathrm{d} \tau \\
& \geqslant \int_{0}^{t} \dot{v}_{i}(\tau)(M v(\tau))_{i} \mathrm{~d} \tau-\left\|\int_{0}^{t} \dot{v}(\tau) \mathrm{d} \tau\right\|(\mu t\|v(t)\|) \\
& \geqslant \int_{0}^{t} \dot{v}_{i}(\tau)(M v(\tau))_{i} \mathrm{~d} \tau-\mu t\|v(t)\|^{2}
\end{aligned}
$$

for all $t \in[0, \varepsilon]$ and all $i=1, \ldots, m$. If $M$ is of type II, then $\sigma_{v}>0$ and $\varepsilon_{v}>0$ exist such that

$$
0 \geqslant \max _{1 \leqslant i \leqslant m}\left\{\int_{0}^{t} \dot{v}_{i}(\tau)(M v(\tau))_{i} \mathrm{~d} \tau\right\}-\mu t\|v(t)\|^{2} \geqslant\left(\sigma_{v}-\mu t\right)\|v(t)\|^{2} \quad \forall t \in\left[0, \varepsilon_{v}\right]
$$


Thus for all $t>0$ sufficiently small, $v(t)=0$ which further implies $u(t)=0$ for almost all such $t$ by the non-negativity and integrability of $u(t)$. It $M$ of type I, then $\sigma_{v}$ and $\varepsilon_{v}$ are both independent of $v$; hence, assertion (b) holds.

\section{A CLASS OF LCSs}

We consider the $\operatorname{LCS}\left(A, B, C, 0, x^{0}\right)$, i.e. the system

$$
\begin{aligned}
\dot{x}(t) & =A x(t)+B u(t) \\
0 & \leqslant u(t) \perp C x(t) \geqslant 0 \\
x(0) & =x^{0}
\end{aligned}
$$

where the initial solution $x^{0}$ satisfies $C x^{0} \geqslant 0$. Since we are interested in solutions with absolutely continuous $x$-trajectories, it follows that any solution to the above LCS must satisfy $C x(t) \geqslant 0$ for all $t$ of interest. Our goal in this section is to establish the existence of a weak solution to (5) under an SSC property of the matrix $C B \in \mathfrak{R}^{m \times m}$ and the uniqueness under matrix class properties introduced in Section 4.

\subsection{Existence of weak solutions}

To show the existence of a weak solution of the LCS (5), we employ a time-stepping scheme and establish the (subsequential) convergence of the discrete-time trajectories as the time step tends to zero. Specifically, consider the following time-stepping scheme for the LCS (5):

$$
\begin{gathered}
x^{h, i+1}=x^{h, i}+h\left[A x^{h, i+1}+B u^{h, i+1}\right] \\
0 \leqslant u^{h, i+1} \perp C x^{h, i+1} \geqslant 0
\end{gathered}
$$

where $h>0$ is the time step and $x^{h, 0}=x(0)$ is the initial condition. A straightforward computation shows that

$$
x^{h, i+1}=(I-h A)^{-1} x^{h, i}+h(I-h A)^{-1} B u^{h, i+1}
$$

for all $h>0$ sufficiently small, therefore, the complementarity condition becomes:

$$
0 \leqslant u^{h, i+1} \perp C(I-h A)^{-1} x^{h, i}+h C(I-h A)^{-1} B u^{h, i+1} \geqslant 0
$$

Suppose that $C B$ is SSC (of type 0). It follows that $C(I-h A)^{-1} B$ is also SSC for all $h>0$ sufficiently small. Thus by LCP theory, the LCP (9) has a solution $u^{h, i+1}$ for all $h>0$ sufficiently small. In general, there are possibly multiple such solutions, each of which induces a vector $x^{h, i+1}$ by (8) that satisfies $C x^{h, i+1} \geqslant 0$. Repeating this argument, we deduce the existence of two sequences of iterates $\left\{x^{h, i}\right\}_{i=0}^{\infty} \subset C^{-1}\left(\mathfrak{R}_{+}^{m}\right)$ and $\left\{u^{h, i}\right\}_{i=1}^{\infty} \subset \mathfrak{R}_{+}^{m}$, from which we construct piecewise functions $\widehat{x}^{h}(t)$ and $\widehat{u}^{h}(t)$ as follows. Let $\hat{x}^{h}(\cdot)$ be the continuous piecewise linear interpolant of the family $\left\{x^{h, i}\right\}$; i.e. for $i=0,1, \ldots, N_{h} \equiv T / h$,

$$
\widehat{x}^{h}(t) \equiv x^{h, i}+\frac{t-t_{h, i}}{h}\left(x^{h, i+1}-x^{h, i}\right) \quad \forall t \in\left[t_{h, i}, t_{h, i+1}\right]
$$

let $\widehat{u}^{h}(\cdot)$ be the (possibly discontinuous) piecewise constant interpolant of the family $\left\{u^{h, i}\right\}$, i.e. $\widehat{u}^{h}(t) \equiv u^{h, i+1}$ for $t \in\left(t_{h, i}, t_{h, i+1}\right]$. It is clear that $C \widehat{x}^{h}(t) \geqslant 0$ for all $h>0$ sufficiently small and all 
$t \in[0, T]$. The result below asserts the convergence of these discrete-time trajectories $\left(\widehat{x}^{h}, \widehat{u}^{h}\right)$ to a weak solution of the LCS (5). (A note about the proof: while there is a detailed investigation of the convergence of time-stepping schemes such as (6)-(7) in [23], the results therein are not directly applicable here because the imposed SSC assumption is weaker than the assumptions used in the reference; nevertheless, the basic line of proof is available and we only need to verify certain key assumptions established in the reference.)

\section{Theorem 7}

Suppose that $C B$ is SSC. The following statements hold:

(a) A scalar $\bar{h}>0$ exists such that the iterates $\left\{x^{h, i}\right\}$ and $\left\{u^{h, i}\right\}$, and thus the pair $\left(\widehat{x}^{h}, \widehat{u}^{h}\right)$, are all well defined for all $h \in(0, \bar{h}]$.

(b) There is a sequence $\left\{h_{v}\right\} \downarrow 0$ such that the following two limits exist: $\widehat{x}^{h_{v}} \rightarrow \widehat{x}$ uniformly on $[0, T]$ and $\widehat{u}^{h_{v}} \rightarrow \widehat{u}$ weakly in $L^{2}(0, T)$.

(c) All limits $(\widehat{x}, \widehat{u})$ obtained in (b) are weak solutions of (5).

Proof

According to [23, Lemma 7.2 and Theorem 7.1], it suffices to show that there are positive constants $h_{1}, \rho_{u}$, and $\psi_{x}$ such that for all $h \in\left(0, h_{1}\right)$ and all non-negative integers $i$ with $(i+1) h \leqslant T$,

$$
\begin{gathered}
\left\|u^{h, i+1}\right\| \leqslant \rho_{u}\left(1+2\left\|x^{h, i}\right\|\right) \\
\left\|x^{h, i+1}-x^{h, i}\right\| \leqslant h \psi_{x}\left(1+\left\|x^{h, i}\right\|\right)
\end{gathered}
$$

We show this using the characterization (3) of strict semicopositivity. First, a scalar $h_{0}>0$ exists such that for all $h \in\left(0, h_{0}\right]$

$$
\max _{1 \leqslant i \leqslant m} u_{i}\left[C(I-h A)^{-1} B u\right]_{i} \geqslant \sigma\|u\|^{2} \quad \forall u \in \mathfrak{R}_{+}^{m}
$$

From (9) and the above bound, we deduce

$$
\begin{aligned}
\left\|u^{h, i+1}\right\|^{2} & \leqslant \frac{-1}{\sigma h}\left(u^{h, i+1}\right)^{\mathrm{T}} C(I-h A)^{-1} x^{h, i} \\
& =\frac{-1}{\sigma h}\left(u^{h, i+1}\right)^{\mathrm{T}} C x^{h, i}+\frac{1}{\sigma h}\left(u^{h, i+1}\right)^{\mathrm{T}} C\left[I-(I-h A)^{-1}\right] x^{h, i} \\
& \leqslant \frac{-1}{\sigma}\left(u^{h, i+1}\right)^{\mathrm{T}} C(I-h A)^{-1} A x^{h, i} \quad \text { because } u^{h, i+1} \geqslant 0 \text { and } C x^{h, i} \geqslant 0
\end{aligned}
$$

Consequently, (10) holds for some constant $\rho_{u}$ independent of $h$. From (6), we obtain

$$
\left\|x^{h, i+1}-x^{h, i}\right\| \leqslant h\left\|(I-h A)^{-1}\right\|\left[\left\|B u^{h, i+1}\right\|+\left\|A x^{h, i}\right\|\right]
$$

Thus, (11) follows from (10).

We are aware of the similarity between the above theorem and the approximation result for LCSs in [28]. However, there exist several major differences: the LCS considered in [28] possesses either a semidefinite $D$ or the passivity property for the matrix tuple $(A, B, C, D)$ and admits a discontinuous state trajectory $x(t)$; on the other hand, the LCS treated in Theorem 7 has a zero $D$ and an absolutely continuous state trajectory $x(t)$ is considered. 


\subsection{Solution boundedness and uniqueness}

In this subsection, we establish two properties of a solution trajectory of the LCS (5); the first property is that any such trajectory must be bounded by a time-dependent multiplicative function times the norm of the initial condition; the second property is 'short-time uniqueness' of any solution trajectory under a restriction on the initial condition. The following lemma provides the key for both properties.

\section{Lemma 8}

If $(x(t), u(t))$ is any solution trajectory of the LCS (5), then $u$ must satisfy

$$
0 \leqslant u(t) \perp C \mathrm{e}^{A t} x^{0}+C B \int_{0}^{t} u(\tau) \mathrm{d} \tau+\Phi(t, u) \geqslant 0
$$

for almost all $t \in[0, T]$, where $\Phi(t, u)$ is absolutely continuous in $t$ for fixed $u \in \mathrm{I}^{m}[0, T]$ and satisfies the following condition: there exist positive constants $\left(\mu, \varepsilon^{\prime}\right)$, which depend only on the triple $(A, B, C)$, such that $\|\Phi(t, u)\| \leqslant \mu t\left\|\int_{0}^{t} u(\tau) \mathrm{d} \tau\right\|$ for all $t \in\left[0, \varepsilon^{\prime}\right]$ and all $u \in \mathrm{I}_{+}^{m}\left[0, \varepsilon^{\prime}\right]$.

Proof

Since $x(t)$ is absolutely continuous on $[0, T]$, it is differentiable at almost all $t \in[0, T]$. Hence so is $\mathrm{e}^{-A t} x(t)$. We have

$$
\begin{aligned}
\frac{\mathrm{d}\left(\mathrm{e}^{-A t} x(t)\right)}{\mathrm{d} t} & =-A \mathrm{e}^{-A t} x(t)+\mathrm{e}^{-A t} \dot{x}(t)=-A \mathrm{e}^{-A t} x(t)+\mathrm{e}^{-A t}[A x(t)+B u(t)] \\
& =\mathrm{e}^{-A t} B u(t)
\end{aligned}
$$

Hence,

$$
\mathrm{e}^{-A t} x(t)-x^{0}=\int_{0}^{t} \frac{\mathrm{d}\left(\mathrm{e}^{-A \tau} x(\tau)\right)}{\mathrm{d} \tau} \mathrm{d} \tau=\int_{0}^{t} \mathrm{e}^{-A \tau} B u(\tau) \mathrm{d} \tau
$$

Consequently, we have

$$
x(t)=\mathrm{e}^{A t} x^{0}+\int_{0}^{t} \mathrm{e}^{A(t-\tau)} B u(\tau) \mathrm{d} \tau
$$

Based on this, we further have

$$
\begin{aligned}
C x(t) & =C \mathrm{e}^{A t} x^{0}+C \mathrm{e}^{A t} \int_{0}^{t} \mathrm{e}^{-A \tau} B u(\tau) \mathrm{d} \tau \\
& =C \mathrm{e}^{A t} x^{0}+C\left[I+\left(\mathrm{e}^{A t}-I\right)\right] \int_{0}^{t}\left[I+\left(\mathrm{e}^{-A \tau}-I\right)\right] B u(\tau) \mathrm{d} \tau \\
& =C \mathrm{e}^{A t} x^{0}+C B \int_{0}^{t} u(\tau) \mathrm{d} \tau+\Phi(t, u)
\end{aligned}
$$

where

$$
\Phi(t, u)=C\left(\mathrm{e}^{A t}-I\right) \int_{0}^{t} \mathrm{e}^{-A \tau} B u(\tau) \mathrm{d} \tau+C \int_{0}^{t}\left(\mathrm{e}^{-A \tau}-I\right) B u(\tau) \mathrm{d} \tau
$$

It is obvious that $\Phi(t, u)$ is absolutely continuous in $t$ for fixed $u \in \mathrm{I}^{m}[0, T]$. Note that there exist $\varepsilon^{\prime}>0, c_{1}>0$, and $c_{2}>0$ such that $\left\|\mathrm{e}^{A t}-I\right\| \leqslant c_{1} t$ and $\max _{i, j}\left|\left[\left(\mathrm{e}^{-A t}-I\right) B\right]_{i j}\right| \leqslant c_{2} t$ for all $t \in\left[0, \varepsilon^{\prime}\right]$. 
Letting $c_{3} \equiv \max _{i, j}\left(\max _{t \in\left[0, \varepsilon^{\prime}\right]}\left|\left(\mathrm{e}^{-A t} B\right)_{i j}\right|\right)$ and recalling that $B$ is of order $n \times m$, we deduce by the non-negativity of $u(t)$ that for all $t \in\left[0, \varepsilon^{\prime}\right]$,

$$
\left\|\int_{0}^{t} \mathrm{e}^{-A \tau} B u(\tau) \mathrm{d} \tau\right\| \leqslant c_{3} \sqrt{n}\left\|\int_{0}^{t} u(\tau) \mathrm{d} \tau\right\|
$$

and

$$
\left\|\int_{0}^{t}\left(\mathrm{e}^{-A \tau}-I\right) B u(\tau) \mathrm{d} \tau\right\| \leqslant c_{2} \sqrt{n} t\left\|\int_{0}^{t} u(\tau) \mathrm{d} \tau\right\|
$$

Therefore, for some constant $\mu>0$ dependent only on the matrices $(A, B, C)$ and not on the solution $u$, we have all $t \in\left[0, \varepsilon^{\prime}\right]$,

$$
\begin{aligned}
\|\Phi(t, u)\| & \leqslant c_{t} t\|C\|\left\|\int_{0}^{t} \mathrm{e}^{-A \tau} B u(\tau) \mathrm{d} \tau\right\|+\|C\|\left\|\int_{0}^{t}\left(\mathrm{e}^{-A \tau}-I\right) B u(\tau) \mathrm{d} \tau\right\| \\
& \leqslant \mu t\left\|\int_{0}^{t} u(\tau) \mathrm{d} \tau\right\|
\end{aligned}
$$

This establishes the desired property of $\Phi(t, u)$. Note that $\varepsilon^{\prime}$ is also independent of the solution $u$.

Without the term $\Phi(t, u)$, expression (12) is precisely the DCP $(q, C B, t)$, where $q(t)=C \mathrm{e}^{A t} x^{0}$. Thus, Lemma 8 clearly brings out the connection between the LCS and DCP; specifically, the DCP is like an integral form of the LCS, albeit including an auxiliary term. Due to the latter term, the existence of a solution to the LCS (established in Theorem 7) does not follow from Mandelbaum's result mentioned in Section 2.

To present the main boundedness and uniqueness properties of the solutions of the LCS (5), we review some basic notions. An ordered tuple $a \equiv\left(a_{1}, \ldots, a_{k}\right)$ of real numbers is said to be lexicographically non-negative if either $a=0$ or its first non-zero component is positive. In this case, we write $a \succcurlyeq 0$. The set of lexicographically non-negative $k$-tuples forms a convex, albeit not closed, cone in $\mathfrak{R}^{k}$. A finite collection of $n$-dimensional vectors $\left(y^{1}, y^{2}, \ldots, y^{k}\right)$ for some positive integer $k$ is said to be lexicographically non-negative, denoted $\left(y^{1}, y^{2}, \ldots, y^{k}\right) \succcurlyeq 0$, if for each $j=1, \ldots, n$, the $k$-dimensional tuple $\left(y_{j}^{1}, \ldots, y_{j}^{k}\right)$ is lexicographically non-negative. Using this notation, the semiobservability cone [19] associated with the pair $(C, A)$ is defined as

$$
\mathscr{Y} \equiv\left\{x \in \mathfrak{R}^{n}:\left(C x, C A x, \ldots, C A^{n-1} x\right) \succcurlyeq 0\right\}
$$

This cone has played a fundamental role in the study of the LCS and its conewise generalization [18]. For our purpose here, the key property we will make use of the cone $\mathscr{Y}$ is the fact that $x^{0} \in \mathscr{Y}$ if and only if $C \mathrm{e}^{A t} x^{0} \geqslant 0$ for all $t \geqslant 0$ sufficiently small; in particular, we must have $C x^{0} \geqslant 0$. Also of significance in the result below is the well-known unobservable subspace associated with the same pair:

$$
\bar{O}(C, A) \equiv\left\{x \in \mathfrak{R}^{n}: C A^{i} x=0, i=0, \ldots, n-1\right\}=\mathscr{Y} \cap(-\mathscr{Y})
$$

It is clear that if $x^{0} \in \bar{O}(C, A)$, then we must have $C \mathrm{e}^{A t} x^{0}=0$ for all $t \geqslant 0$. The following is the main result for the LCS (5). In the result, we write $\left(x\left(t, x^{0}\right), u\left(t, x^{0}\right)\right)$ for a solution trajectory to the LCS in order to stress the dependence of the trajectory on the initial condition $x^{0}$. 
Theorem 9

The following three statements hold for the LCS (5).

(a) If $C B$ is SSC of type I, then there exist scalars $\varepsilon_{1}>0, \sigma_{2}>0$ and a function $\sigma_{1}(t)>0$ such that for all initial states $x^{0}$ satisfying $C x^{0} \geqslant 0$ and all $t \in\left[0, \varepsilon_{1}\right]$, any solution $\left(x\left(t, x^{0}\right)\right.$, $\left.u\left(t, x^{0}\right)\right)$ of the $\operatorname{LCS}(5)$ satisfies

$$
\left\|x\left(t, x^{0}\right)\right\| \leqslant \sigma_{1}(t)\left\|x^{0}\right\| \quad \text { and } \quad\left\|\int_{0}^{t} u\left(\tau, x^{0}\right) \mathrm{d} \tau\right\| \leqslant \sigma_{2}\left\|x^{0}\right\|
$$

(b) If $C B$ is SSC of type I, then there exists a scalar $\varepsilon_{2}>0$ such that for any $x^{0} \in \mathscr{Y}$, there is a unique solution pair $\left(x^{*}\left(t, x^{0}\right), u^{*}\left(t, x^{0}\right)\right)$ of the $\operatorname{LCS}(5)$ in the interval $\left[0, \varepsilon_{2}\right]$ that is given by

$$
\begin{gathered}
x^{*}\left(t, x^{0}\right)=\mathrm{e}^{A t} x^{0} \quad \forall t \in\left[0, \varepsilon_{2}\right] \\
u^{*}\left(t, x^{0}\right)=0 \text { for almost all } t \in\left[0, \varepsilon_{2}\right]
\end{gathered}
$$

Moreover, if $x^{0} \in \bar{O}(C, A)$, then the latter conclusion holds for $\varepsilon_{2}=T$.

(c) If $C B$ is SSC of type II, then for any $x^{0} \in \mathscr{Y}$ and any solution $\left(x\left(t, x^{0}\right), u\left(t, x^{0}\right)\right)$ of the LCS (5), there exists $\varepsilon_{u}>0$ such that $x\left(t, x^{0}\right)=\mathrm{e}^{A t} x^{0}$ for all $t \in\left[0, \varepsilon_{u}\right]$ and $u\left(t, x^{0}\right)=0$ for almost all $t \in\left[0, \varepsilon_{u}\right]$.

\section{Proof}

The proof is based on the integral property (12) of a solution of the LCS. For part (c) and the first assertion in part (b), it suffices to combine this integral representation of a solution with Proposition 6, taking note of the above-mentioned properties of the elements in the cone $\mathscr{Y}$. There is no need for further proof of these two assertions. Based on the first assertion in part (b), the second assertion can be proved as follows. Let $x^{0} \in \bar{O}(C, A) \subseteq \mathscr{Y}$. Let $\varepsilon_{2}>0$ be as prescribed in the first part of (b). Next consider the LCS starting at time $t=\varepsilon_{2}$ with initial condition $x\left(\varepsilon_{2}, x^{0}\right)$ which remains in $\bar{O}(C, A)$. Since the LCS is a time-invariant system and the scalar $\varepsilon_{2}$ is independent of initial conditions and solution trajectories, we can extend the uniqueness of the solution trajectory on hand to the interval $\left[\varepsilon_{2}, 2 \varepsilon_{2}\right]$. Continuing this argument will allow us to reach the final time $T$.

It remains to prove (a). Note that (12) yields

$$
\int_{0}^{t} u\left(s, x^{0}\right) \circ\left[C \mathrm{e}^{A s} x^{0}+C B v\left(s, x^{0}\right)+\Phi\left(s, u\left(\bullet, x^{0}\right)\right)\right] \mathrm{d} s=0
$$

for all $t \in[0, T]$, where $v\left(t, x^{0}\right) \equiv \int_{0}^{t} u\left(\tau, x^{0}\right) \mathrm{d} \tau$ and $\Phi\left(t, u\left(\bullet, x^{0}\right)\right)$ satisfies

$$
\left\|\Phi\left(t, u\left(\bullet, x^{0}\right)\right)\right\| \leqslant \mu t\left\|v\left(t, x^{0}\right)\right\|
$$

for all $t \in\left[0, \varepsilon^{\prime}\right]$, with the constants $\mu$ and $\varepsilon^{\prime}$ being independent of the $x^{0}$ and solution pairs. Similar to the proof of Proposition 6, we deduce that for some index $i$ and constants $\sigma^{\prime} \in(0, \sigma)$ and $\varepsilon$ satisfying $0<\varepsilon<\min \left(\bar{t}, \mu^{-1}\left(\sigma-\sigma^{\prime}\right)\right)$, where $\sigma$ and $\bar{t}$ are two positive constants associated with the matrix type I,

$$
\sigma^{\prime}\left\|v\left(t, x^{0}\right)\right\|^{2} \leqslant(\sigma-\mu t)\left\|v\left(t, x^{0}\right)\right\|^{2} \leqslant\left|\int_{0}^{t} \dot{v}_{i}\left(\tau, x^{0}\right)\left(C \mathrm{e}^{A \tau} x^{0}\right)_{i} \mathrm{~d} \tau\right| \quad \forall t \in[0, \varepsilon]
$$


Moreover, we have, for some constant $\kappa>0$ that depends only on $(A, B, C)$,

$$
\begin{aligned}
\left|\int_{0}^{t} \dot{v}_{i}\left(\tau, x^{0}\right)\left(C \mathrm{e}^{A \tau} x^{0}\right)_{i} \mathrm{~d} \tau\right| & \leqslant \int_{0}^{t} \dot{v}_{i}\left(\tau, x^{0}\right)\left|\left(C \mathrm{e}^{A \tau} x^{0}\right)_{i}\right| \mathrm{d} \tau \leqslant \int_{0}^{t} \dot{v}_{i}\left(\tau, x^{0}\right)\left\|C \mathrm{e}^{A \tau} x^{0}\right\| \mathrm{d} \tau \\
& \leqslant \kappa\left\|x^{0}\right\| \int_{0}^{t} \dot{v}_{i}\left(\tau, x^{0}\right) \mathrm{d} \tau \leqslant \kappa\left\|x^{0}\right\|\left\|v\left(t, x^{0}\right)\right\|
\end{aligned}
$$

Consequently, for some constant $\sigma_{2}>0$, we obtain

$$
\left\|v\left(t, x^{0}\right)\right\| \leqslant \sigma_{2}\left\|x^{0}\right\| \quad \forall t \in[0, \varepsilon]
$$

From (13), we deduce, for some constant $c>0$,

$$
\left\|x\left(t, x^{0}\right)\right\| \leqslant\left\|\mathrm{e}^{A t}\right\|\left[\left\|x^{0}\right\|+\left\|\int_{0}^{t} \mathrm{e}^{-A \tau} B u\left(\tau, x^{0}\right) \mathrm{d} \tau\right\|\right] \leqslant\left\|\mathrm{e}^{A t}\right\|\left[\left\|x^{0}\right\|+c\left\|v\left(t, x^{0}\right)\right\|\right]
$$

from which the existence of the function $\sigma_{1}(t)$ follows readily.

It should be pointed out that while the existence of a weak solution to the LCS (5) is established for any finite time $T>0$ (Theorem 7), the solution properties are valid only on short-time intervals (Theorem 9). The difficulty in extending the latter properties to long time intervals is twofold. One, while the integral formulation (12) of the LCS is valid for any time $t>0$, the key bound $\| \Phi(t, u)$ $\|\leqslant \mu t\| v(t) \|$ is valid only for a short time. Two, the condition $x^{0} \in \mathscr{Y}$ ensures that $C \mathrm{e}^{A t} x^{0} \geqslant 0$ for a short time only; when some component of the vector $C \mathrm{e}^{A t} x^{0}$ reaches zero at some time $t>0$, a possible jump in the algebraic variable $u\left(t, x^{0}\right)$ occurs and the LCS enters into a new mode. Without a clear understanding about this mode transition, which is particularly challenging because the key matrix $D$ in the LCS $(A, B, C, D)$ is zero in this case, we cannot predict the trajectory behaviour after the event time. Of course, a question remains as to what happens when $x^{0} \notin \mathscr{Y}$; at this time, we have no answer for this question. However, to illustrate the non-triviality of this question, we present the following example, which is originally proposed in [25] and recently revisited in [22].

Example 10

Consider the index-one $\operatorname{LCS}(A, B, C, 0)$ with

$$
A=\left[\begin{array}{cc}
0 & -1 \\
0 & 0
\end{array}\right] \in \mathfrak{R}^{4 \times 4}, \quad B=\left[\begin{array}{l}
R \\
0
\end{array}\right] \in \mathfrak{R}^{4 \times 3} \quad \text { and } \quad C=\left[\begin{array}{ll}
I_{3} & 0
\end{array}\right] \in \mathfrak{R}^{3 \times 4}
$$

where $\mathbf{1}=\left[\begin{array}{lll}1 & 1 & 1\end{array}\right]^{\mathrm{T}}$ and $R$ is the non-negative $P$-matrix given by

$$
R=\left[\begin{array}{lll}
1 & 3 & 0 \\
0 & 1 & 3 \\
3 & 0 & 1
\end{array}\right]
$$

It is clear that $C B=R$ is a $P$-matrix. However, if $x^{0}=(0,0,0,1)$ so that $x^{0} \notin \mathscr{Y}$, then the LCS can be cast as $\operatorname{DCP}(-1 t, R)$ : find an integrable $u(t)$ such that

$$
0 \leqslant u(t) \perp-1 t+R \int_{0}^{t} u(\tau) \mathrm{d} \tau \geqslant 0 \text { for almost all } t \geqslant 0
$$

It is shown in [25] that the latter DCP admits multiple solutions and thus uniqueness fails. Nevertheless, if the solution space is restricted to be piecewise Bohl distributions, then there is a 
unique Bohl-type solution since $C B$ is a $P$-matrix. Moreover, since $C B$ is a non-negative matrix with positive diagonal entries, thus is SSC of type I, it follows 'short-time' solution uniqueness holds for any $x^{0} \in \mathscr{Y}$.

\section{ACKNOWLEDGEMENTS}

The authors are grateful to Professor David Stewart at the University of Iowa for many constructive comments regarding this paper and to the referees for their very carefully reading and checking the first version of the paper. In particular, a referee kindly reminded us of Example 10. Stewart's preprint [22] is an inspiration for the revision of our paper.

\section{REFERENCES}

1. Cottle RW, Pang JS, Stone RE. The Linear Complementarity Problem. Academic Press: Cambridge, 1992.

2. Facchinei F, Pang JS. Finite-Dimensional Variational Inequalities and Complementarity Problems I. Springer: New York, 2003.

3. Çamlibel MK. Complementarity methods in the analysis of piecewise linear dynamical systems. Ph.D. Thesis, Center for Economic Research, Tilburg University, The Netherlands, 2001.

4. Çamlıbel MK, Schumacher JM. Existence and uniqueness of solutions for a class of piecewise linear systems. Linear Algebra and its Applications 2002; 351-352:147-184.

5. Heemels WPH. Linear complementarity systems: a study in hybrid dynamics. Ph.D. Thesis, Department of Electrical Engineering, Eindhoven University of Technology, November 1999.

6. Heemels WPMH, Schumacher JM, Weiland S. Well-posedness of linear complementarity systems. Proceedings of the 38th IEEE Conference on Decision and Control, Phoenix, U.S.A., 1999; 3037-3042.

7. Heemels WPMH, Schumacher JM, Weiland S. The rational linear complementarity systems. Linear Algebra and its Applications 1999; 294:93-135.

8. Heemels WPMH, Schumacher JM, Weiland S. Linear complementarity systems. SIAM Journal on Applied Mathematics 2000; 60:1234-1269.

9. Schumacher JM. Complementarity systems in optimization. Mathematical Programming, Series B 2004; 101: $263-295$.

10. van der Schaft AJ, Schumacher JM. The complementarity-slackness class of hybrid systems. Mathematics of Control, Signals, and Systems 1996; 9:266-301.

11. van der Schaft AJ, Schumacher JM. Complementarity modeling of hybrid systems. IEEE Transactions on Automatic Control 1998; 43:483-490.

12. Mandelbaum A. The dynamic complementarity problem. Unpublished manuscript, Technion, 1989.

13. Çamlıbel MK, Heemels WPMH, Schumacher JM. On linear passive complementarity systems. European Journal of Control 2002; 8:220-237.

14. Çamlıbel MK. Popov-Belevich-Hautus type controllability tests for linear complementarity systems. Systems and Control Letters 2007, to appear.

15. Çamlıbel MK, Heemels WPMH, Schumacher JM. Algebraic necessary and sufficient conditions for the controllability of conewise linear systems. IEEE Transactions on Automatic Control 2007, in press.

16. Çamlıbel MK, Heemels WPMH, Schumacher JM. On the controllability of bimodal piecewise linear systems. In Hybrid Systems: Computation and Control, Alur A, Pappas GJ (eds). Lecture Notes on Control and Systems 2993. Springer: Berlin, 2002; 250-264.

17. Çamlıbel MK, Pang JS, Shen J. Lyapunov stability of complementarity and extended systems. SIAM Journal on Optimization 2006; 17(4):1056-1101.

18. Çamlıbel MK, Pang JS, Shen J. Conewise linear systems: non-Zenoness and observability. SIAM Journal on Control and Optimization 2006; 45(6):1769-1800.

19. Pang JS, Shen J. Strongly regular differential variational systems. IEEE Transactions on Automatic Control 2007, in press.

20. Pang JS, Stewart D. Solution dependence on initial conditions in differential variational inequalities. Mathematical Programming, Series B 2007, in press.

21. Shen J, Pang JS. Linear complementarity systems: Zeno states. SIAM Journal on Control and Optimization 2005; 44:1040-1066. 
22. Stewart DE. Uniqueness for index-one differential variational inequalities. Manuscript, Department of Mathematics, University of Iowa, Iowa City, October 2006.

23. Pang JS, Stewart DE. Differential variational inequalities. Mathematical Programming, Series A 2007, in press.

24. Stewart DE. Convolution complementarity problems with application to impact problems. IMA Journal of Applied Mathematics 2006; 71:92-119.

25. Bernard A, El Kharroubi A. Régulations de processus dans le premier 'orthant' de $\mathfrak{R}^{n}$. Comptes Rendus Academie Science Paris Series I Mathematique 1989; 309:371-375.

26. Royden HL. Real Analysis (2nd edn). Macmillan: New York, 1968.

27. Folland GB. Real Analysis: Modern Techniques and Their Applications (2nd edn). Wiley: New York, 1999.

28. Camlibel MK, Heemels WPMH, Schumacher JM. Consistency of a time-stepping method for a class of piecewise linear networks. IEEE Transactions on Circuit and Systems 2002; 49(3):349-357. 